\title{
After Fidelity: Deconstructionist Translation Theory \& Deconstruction of Cultural Translation
}

\author{
Wanfang Zhang \\ School of Foreign Languages \\ Wuhan Polytechnic University \\ Wuhan, China \\ 420300466@qq.com
}

\author{
Xiangxin Liu \\ Department of Public Relations \\ Wuhan Railway Vocational Technological University \\ Wuhan, China \\ 1287958075@qq.com
}

\begin{abstract}
When talking about deconstructionist translation theory, quite a few people consider it to be against the traditional standard "Fidelity". And this thesis is trying to tell the connection between the two and how one can adopt the theory when conducting translation. This thesis will inspire its readers to break the normal thinking modes and to try new and creative ways in translation.
\end{abstract}

Keywords-deconstructionist translation theory; fidelity; cultural transformation; cultural translation

\section{INTRODUCTION}

The purpose of this paper is trying to give its readers the definition and development of deconstructionist translation theory, the theory's connections with the traditional standard "Fidelity", the positive and negative influences in the translation field, and how to translate the cultural works when using deconstructionist translation theory. Lots of examples of cultural translation deconstruction will be listed in the paper in order to make the paper more specific, thus making it of more practical value. When talking about deconstructionist translation theory, quite a few people consider it to be against the traditional standard "Fidelity". And this thesis is trying to tell the connection between the two and how one can adopt the theory when conducting translation. There is no doubt that this thesis is of value to deconstructionist translation theory learning and using. The second part mainly deals with the deconstruction of cultural translation. Besides, the theory overcomes the Saussure's structuralism, which rules translation for many years. This thesis will inspire its readers to break the normal thinking modes and to try new and creative ways in translation.

\section{FIDELITY AND THE DECONSTRUCTIONIST TRANSLATION THEORY}

\section{A. "Fidelity" in the Traditional Translation Standards}

Translation refers to the transfer of meanings, structures, modes etc. between different languages. There surely exist blind points in correspondences between the two languages in morphology, syntax, and ways of writing or expressing, among many others. Fidelity is deemed the first criterion of translation.

No matter in west or east, the traditional translation standards all stress the "fidelity" criterion. Translation must be proceed on the basis of comprehending what author want to convey to others and meanwhile explicitly give an intact presentation of it in another language, which decides that translator must attempt convey the author's thought accurately. In China, scholars are mostly in favor of Yan Fu's standard of translation, namely "faithfulness, expressiveness and elegancy". The faithfulness, in other words, is fidelity. And the fact that this standard has come all the way to modern translation academy indeed has its own meaning. But fidelity does not implicate inflexible or mechanical equivalent in meaning; absolute faithfulness cannot be achieved either.

Different ideas about fidelity are given by many notable translators. Such as Lu Xun, in his book A Collection of Foreign Fictions-Preface in 1909, presents an idea of literal translation and later developed it in his translation practice, with a characteristic translation style gradually in shape. About fidelity, Lu Xun said that "faith rather than fluency" should be put into practice in translation process, on the other side, he said "if only to seek for understandability, you can choose to take a creation of it" (Lu Xun, 1981). And he states that even in literary translation, literal translation should be chosen to keep the features of original text. Obviously, Lu Xun maintains foreinization not domestication. For instance he himself took on the issue of foreignization, when he translated one foreign sentence as one unsmooth version-- "shan bei hou tai yang xia qu le" rather than "Riluoxishan" for he maintains that the original text focuses on the mountain, if changed, the focus shifts to the sun.

Lin Yutang, in his book On Translation, puts forward one set of standards-faithfulness, fluency and beauty. His understanding of faithfulness can be flexible to a large extend. And he gives four definitions about faithfulness: first, faithfulness is based on the "total image" not the literal meaning of word. Thus, it is sentence translation rather than word-to-word translation that can achieve fidelity; second, there is no possibility of absolute faithfulness; third, fidelity requires not only expressing ideas of the author's but also being vivid or lifelike, in other words, taking the implicated meaning out the text so as to make the text be known; forth, faithfulness should not be opaque in Chinese, so he is in favor of domestication and translating according to Chinese writing style. As we can see, the two figures above hold different point in the understanding of fidelity in translation standard. What causes this is their different pursue of the value of translation, yet this is not discussed here. 
On the basis of Yan Fu's “xin-da-ya”, many other scholars, such like Liu Chongde (xin-da-qie), Fu Lei (sprit likeness), Qian Zhongshu (transmigrations), Xu Yuanchong (xin-da-you), develop their own standards for translation mostly with a change of the "ya" namely "elegance" put forward by Yan Fu. And this paper gives its own understanding into fidelity.

Fidelity, to start with, is to convey original text content accurately and honestly, with no distortion of it or omission at will. The content not only refers to the facts, truths, scenery described in the text, but also the thought, standing point and feelings the text reflects. Besides, fidelity also refers to the similarity in writing style in both the two languages. Here, the standard fidelity is not the traditional one, some new elements having been put into it. Translator, serving both the author and reader, must be loyal to the readers while he finds fidelity in translation. Because if not, readers cannot understand, further accept the translation; then there is little possibility for the translation to have the communication goal achieved, let alone its value of reading.

In order to make its reader get a throughout knowing about the standard fidelity, the thesis also distinguishes the standard fidelity and the standard accuracy. The fidelity standard in translation calls for an unchanging of sentence's meaning, no overmuch omission, if there are some figures of speeches, better translating it with the same one to get the original beauty conveyed explicitly to the readers. However, accuracy standard is a higher use in diction, or precisely speaking, in figure of speech. We can consider the former one as an overall translation standard, the latter part standard. For students who are beginners in learning translation, the former one is often the basic requirements.

But in reality, fidelity is an ideal standard for translation practice. And at modern times, the fidelity seems gradually vanishing in people's eyes according to some scholars. The polysystem theory states that fidelity seems to blank in translation; the Teleology theory says that the fidelity standard seems to have elasticity (the requirements for fidelity must in accordance to the goal of translation. Given that there are various different goals, the fidelity also has many different definitions and proposes different requirements in translation).

\section{B. Fidelity and the Deconstructionist Translation Theory}

Why and how can the standard "fidelity" for translation last so long in the translation academy? Even now, inevitably, we still consider it as the basic standard when conducting translation teaching. In the west, as early as early as late Roman times, the famous Bible translator Jerome thought the translation should distinguish between two categories: literary translation and religious translation (at that time, especially Bible translation). And toward literary translation, quite a few scholars held the opinion that fidelity standard must be required so as to convey both the textual meanings and writing style to readers. The traditional translation theory has been centered on the emphasis of structure of sentences, text, in other words, of a linguistic way in translation work.

The most prominent feature of the traditional standard "fidelity" is the hallowed identification of original text author thus the emphasis on translator's compliance to the author in translation, from which the fidelity complex comes. There are three pairs of basic relationships in translation: author and translator, SLT (source language text) and TLT (target language text), and SLT readers and VLT readers. The traditional translation studies have been always centered on the former one, taking the former one as the measurement for translation quality; thus scholars neglected other factors outside text, like the goal of translation, its value, its readers, and things like such. The standard fidelity just based on the text, on the language level, can hardly reflect the real phenomena in translation.

Just like all theories are moving forward in history, there is no exception of fidelity. Progress has been made in the translation standard studies which have thrown of the fragmentary empirical and vagrant theories and go to the philosophical height. The studies have gone through a big jump in the shifts from ambiguity, abstraction to systematicness, presicion and operability. Though changes pervade in the translation field, the standards still are the constraints of a particular language or some specific versions.

Influenced by the deconstruction theory, lots of translation studies have been overthrown because the theory's challenge to the basic concepts held by construction theory, such as Logocentrism, dualism, etc. Working under the philosophy guideline of deconstruction, deconstructionist translation theory strike the translation studies with a brand new way of thinking, a totally different position of view (eg: difference and diversity) into the translation problems, which brings us a new way different from the traditional one in describing, analyzing, assessing the translation work. It makes the translation "transferred from the pure-language-level reference translation to a more dynamic cultural interpretation and reappearance” .

The deconstructionist translation theory states that the fidelity is a historical construction rather than natural relations with translation. And it promotes a further development for the translation standards, especially the fidelity. Here, the paper demonstrates the development of fidelity under the influence of deconstructionist translation theory.[2]

To start with, the standard "fidelity" should be comprehensive but focused. The definition of fidelity in translation has changed a lot (see above), and it covers a more extensive factors which is explicitly required in translation. Besides the original factors involved in fidelity, like the text meaning, writing style, figure of speech, other out-of-text factors must also be considered, only by which can the fidelity (now) can be reached. Such factors like people's interest, social translation trend, political climate, publish and market polices, among many other things.

Second, the translation ways like literal translation and free translation must be integrated in "fidelity". Some scholars are against literal translation for it destroys the beauty of the target text, while we should get the essentiality-literal translation itself is not an extreme of translational ways; only when it goes to extreme, the natural beauty of versions will be damaged.

Third, the deconstructionist translation theory places an emphasis on the translator's subjectivity, and so the "fidelity" is relevant to the translator's play of subjectivity. Traditional 
fidelity in translation is somehow an ideal model for translator in translation practice for translation concerns many factors including the linguistic-oriented translation studies' linguistics factors in textual meaning. The difference in culture and languages and the translator subjectivity in translation cannot be avoided, in the case of which absolute fidelity cannot be achieved in translation work. Today, faithfulness is not only a problem in the comparison of textual meaning, but also involves something like reader's demand, translation target, market demand, especially the translator's subjectivity and translational style. Considering many external textual factors, Derrida considers the translation as an economical concept, a human-participant construction and social conventional event. So it is better to have a coordinated relationship between translator and author, the target language culture and original language culture. [3]

Some scholars comprehend some concepts of the deconstructionist translation theory to the extreme that they finally fall into nihilism. Misunderstanding of some scholars into deconstructionist translation theory lead to their disbelief in the translation standard "fidelity" but rather than distrust fidelity, it is better to say that we distrust the authenticity and impartiality of a particular translation standard. Precisely, "deconstruction theory is the destruction of the original text and its author not the denial of the two. The deconstruction theory just comes out of the framework of the emphasis of the original text of the traditional translation standards and gets a lot other external textual factors in sight-seeing more of the full view of translation.” [4].

The respect to the original text and its author and its source does not mean blind fidelity to the source text. When scholars come out of the framework of the traditional structure translation, they can get to know the space-formula interaction between translator, reader, and the version and that the translator's task shouldn't be limited to only the original text or the target text. About this question, Liu Yameng gives a quite good reply- "it is not annihilation but transform should be made to the traditional standard 'fidelity' so as to endow it with a different but more fair and reasonable structure or function in assessing translation works”.(Liu Yameng,2006)

In translation teaching, it's important to get students across about the whole development of fidelity and make them know that the translator's task is more than just translating but also work as a spreader of culture.

\section{CUltURAL TRANSLATION UNDER THE GUIDANCE OF THE DECONSTRUCTIONIST TRANSLATION THEORY}

\section{A. The Deconstruction of Cultural Translation}

What is cultural translation? Before I write on this topic, the only definition I can think about this is that cultural translation is the translation of literary works, and that's all. In order to have a clear understanding of this topic, a lot of materials on this have been searched during the writing process.

The Wikipedia says "cultural translation represents the practice of translation, which involves cultural differences. Cultural translation can be also defined as a practice whose aim is to present another culture via translation." "Cultural translation is a term which must be also studied through cultural anthropology, a field of anthropology focused on cultural issues among humans.”

An essay in the net DOC88.COM says "cultural translation refers to the study of translation on the background of cultural study. To be more explicitly, it means to have a study on the similarity and individuality of the surface structure and the deep structure of different languages so as to have a throughout discuss of the internal relation and objective laws between culture and translation.”

Zhang Junwei says in his paper about cultural translation from a special perspective-“translation is a transformation between two languages. The translation itself should be considered as a transcultural practical activity; as to the translator, the process decoding of the original text and encoding of the target text not only involves languages but also have the translation core-the understanding and apperception of the world we live in-reflected. And that is the 'cultural turn' in translation studies-cultural translation."

From above, this paper concludes the cultural translation to be a study of translation on the background of culture. That means translation has gone into a period of cultural translation, in other words, a cultural turn.

Edwin Gentzler admits that there is little progress made in cultural translation before deconstruction arose; mostly previous theories focus on the problem of equivalence. He says "the deconstructionists put forward some questions and found their theory on the basis of these. They not only provide but also challenge the question about the basic concept in all theories people have concern." And he says that the deconstructionist thinks "perhaps the translated text writes us and not we the translated texts.” (Edwin Gentzler, 2001)

Benjamin had a question that "is translation produced for those who don't understand the original text? It seems that this explanation can be used to fully demonstrate the hierarchical differences existed in the cultural field...however, any translation that is trying to exert its function as informationconveying, is just conveying something at all, therefore, something unessential. Undoubtedly, this is the symbol of clumsy translation. " [5 ] so according to him, what is a good translation? He admits that "translator's task is to find the particular thought that oriented to the target language, and produce echo with the original text in the thought.” [6]

Here are some examples of good translation.

Advertisement translation (from English to Chinese)

Colgate-GaoLujie; Rejoice-PiaoRou; Head\& ShoulderHaifeisi

Obviously, "Colgate" has no meaning of "clean and spotless, but when translated as "GaoLujie", Colgate gives an implication that "this kind of toothpaste can not only make your teeth clean and spotless, but also make you feel confident." The second and third translation both have nothing to do with the original word, but making people having a vivid experience of using these two shampoos. The translation of coffee Maxwell's advertising commentary "Good to last drop" exquisitely uses the four-character idioms in Chinese cultural 
custom, which better caters the habits and mentality of the Chinese people.

All above examples have violated the traditional standard "fidelity", but under the influence of deconstructionist translation theory, the translation not only fascinating but also conform to each country's culture. In some case, that is the fidelity to the original text meaning.

In a word, deconstruction of cultural translation lies on the change of translation standard, especially "fidelity", the promotion of the translator's and target text's position, and a trend for beauty in cultural translation assessing.

\section{B. The Theory's Impact on the Cultural Translation Practice}

The deconstruction theory has brought a lot of challenge to the translation studies. Its challenge to the position of the original text and the target text, the author and the translator has produced a dramatic change on translation theories. In fact, the deconstruction theory itself focuses on theoretical thus can provide little guidance on translation practice. But the third period of its development, namely foreignizaiton, definitely considers the foreignization as a way for translation. Besides these, the deconstruction is the basic theory of the cultural turn in translation studies.[7]

\section{CONCLUSION}

As the title of the thesis implies, the topic elaborates the standard "fidelity" in the traditional sense. It defines the fidelity in the aspect of text meaning, writing style, and figures of speech. The most important writing part goes to the deconstructionist translation theory's influence and change it brings to the "fidelity" and other traditional standards. After the demonstration of the influence on the fidelity, the thesis goes on to summarize the positive and negative impact the deconstructionist translation theory has on the translation academy. In a word, not only change but also no set of standard it has both brings to the evaluation of the translation work.

Deconstructionist translation theory, in some sense, is the basic theory for the cultural turn in the translation work, thus making the cultural translation an important role in translation which serves as an essential communication ways for different nations.[8] In some cases, the cultural translation has finally found its inward place in translation work by the deconstructionist translation theory. The deconstruction of cultural translation involves the challenge of the traditional translation standard, the promotion of translator's position, the recommendation of beauty in the translation works which is considered as a rebirth of the original text. Then, the thesis illustrates the deconstruction of cultural translation according to the three aspects and the deconstructionist theory's impact of the cultural translation practice.

\section{REFERENCES}

[1] J. Hillis Miller. Fiction and Repetition: Seven English Novels [M].Cambridge, Mass: Harvard University Press, 1982.

[2] Jacques Derrida. Joseph F Graham. Des Tours de Babel [A]. Ithaca, New York: Cornell University Press, 1985.

[3] Jacques Derrida. Structure, Sign and the Humanities Discourse in the Play [Z]. Lecture, American Johns Hopkins University, 1966.

[4] Venuti Lawrence. Rethinking Translation [M].London: Routledge, 1992

[5] Walter Benjamin. The Task of the Translator [A]. Chicago\& London the University of Chicago Press 1923.

[6] Walter Benjamin, Theories of Translation: An Anthology of Essays from Dryden to Derrida [M]. Chicago and London: The University of Chicago Press, 1992, pp. 71-72.

[7] William E.Cain. The Crisis in Criticism [M]. The Johns Hopkins University press, 1987.

[8] Derrida, Jacques. Position [M]. Chicago: University of Chicago Press, 1981. 